\title{
The role of visual working memory (VWM) in the control of gaze during visual search
}

\author{
ANDREW HollingWorth \\ University of Iowa, Iowa City, Iowa \\ AND \\ STEVEN J. LUCK \\ University of California, Davis, California
}

\begin{abstract}
We investigated the interactions among visual working memory (VWM), attention, and gaze control in a visual search task that was performed while a color was held in VWM for a concurrent discrimination task. In the search task, participants were required to foveate a cued item within a circular array of colored objects. During the saccade to the target, the array was sometimes rotated so that the eyes landed midway between the target object and an adjacent distractor object, necessitating a second saccade to foveate the target. When the color of the adjacent distractor matched a color being maintained in VWM, execution of this secondary saccade was impaired, indicating that the current contents of VWM bias saccade targeting mechanisms that direct gaze toward target objects during visual search.
\end{abstract}

The successful completion of almost any goal-directed behavior requires orienting attention efficiently to taskrelevant objects in the world. To write and mail a letter, for example, one must shift one's eyes among several objects (paper, pen, envelope, stamp) as each object is required by the task (Land \& Hayhoe, 2001). Unless an object is already attended or draws attention to itself directly, a visual search operation is required to select the relevant object from among other visible objects. In real-world behavior, this selection is controlled primarily by top-down mechanisms. The bottom-up visual salience of an object is unlikely to be strongly correlated with task relevance (Henderson, Brockmole, Castelhano, \& Mack, 2007), and bottom-up salience is unlikely to change systematically as a function of evolving task demands. Thus, visual cues alone would be insufficient to guide attention and the eyes in real-world tasks. Top-down control over search and selection is required and is likely to be dominant in the absence of salient, transient events that capture attention (Franconeri, Hollingworth, \& Simons, 2005; Yantis \& Jonides, 1984).

One means by which top-down control over attention can be exerted is through an interaction between attention and visual working memory (VWM; Chelazzi, Duncan, Miller, \& Desimone, 1998; Chelazzi, Miller, Duncan, \& Desimone, 1993; Desimone \& Duncan, 1995; Duncan \& Humphreys, 1989; Hollingworth, Richard, \& Luck, 2008; Olivers, Meijer, \& Theeuwes, 2006; Soto, Heinke, Humphreys, \& Blanco, 2005; Soto, Humphreys, \& Heinke, 2006; Woodman, Luck, \& Schall, 2007). ${ }^{1}$ The
VWM system is a set of processes that supports the maintenance of perceptual information from a small number of objects across relatively brief delays and perceptual disruptions (for a review, see Luck, 2008b). In search, VWM can provide top-down control by maintaining the visual properties of the search target, allowing each attended object to be categorized as either the target or a nontarget (Duncan \& Humphreys, 1989). In addition, the maintenance of object properties in VWM can interact with the sensory representation of visible objects that share those properties (Chelazzi et al., 1998; Chelazzi et al., 1993), biasing spatial attention toward the location of a remembered object. Specifically, the biased competition model (Chelazzi et al., 1993; Desimone \& Duncan, 1995) holds that VWM retention involves the sustained activation of a perceptual representation of the remembered object. Subsequent perceptual processing of an object that shares the preactivated features will be facilitated, and that object will come to dominate the sensory response, biasing spatial selection during search toward the location of an object that matches memory (Olivers et al., 2006; Soto et al., 2005). ${ }^{2}$ In this manner, when the pen is required by the task, remembered features of the pen (red, cylindrical, small) could be retrieved from long-term memory (LTM) and activated in VWM. The maintenance of these features in VWM would then bias spatial attention during search toward the location of an object with matching features, such that the pen is efficiently selected from among competing objects and becomes available for use in the task.

A. Hollingworth, andrew-hollingworth@uiowa.edu 
VWM is also used to ensure that, after a potentially useful object has been selected covertly, the eyes successfully arrive at the intended object. Such control is necessary because the saccadic eye movements that bring the eyes from one object to the next are prone to error, with the eyes frequently failing to land on the intended saccade target. Saccade errors occur on as many as $40 \%$ of trials in laboratory studies (Frost \& Pöppel, 1976; Hollingworth et al., 2008; Kapoula, 1985). When the eyes fail to land on the target in a natural scene, other objects are likely to be near the landing position of the eye movement, creating a problem of object correspondence: Which object is the original saccade target? By retaining visual features of the saccade target across the saccade, VWM can serve to resolve this ambiguity (Hollingworth et al., 2008; Richard, Luck, \& Hollingworth, 2008).

Attention and VWM appear to work together during eye movements in the following manner. Prior to a saccade, spatial attention is shifted covertly to the location of the impending saccade (Deubel \& Schneider, 1996; Hoffman \& Subramaniam, 1995). Directing attention to the saccade target object supports the consolidation of perceptual features of that object into VWM (Irwin \& Gordon, 1998; Schmidt, Vogel, Woodman, \& Luck, 2002). These perceptual features are maintained in VWM across the saccade (Henderson \& Hollingworth, 2003; Irwin, 1992; Irwin \& Andrews, 1996). If the eyes fail to land on the saccade target, the VWM representation of the target can be used to trigger a secondary saccade to that object and not to other objects, even if other objects are closer to the saccade landing position (Hollingworth et al., 2008; Richard et al., 2008). Reacquiring the target after an errant saccade is one of the most common forms of search conducted by the visual system during everyday behavior. We make hundreds of thousands of saccades each day, and many of those fail to land on the saccade target, requiring an additional visual search operation to ensure that the eyes are oriented to the intended object.

Support for the general claim that the content of VWM guides the allocation of attention during search comes from recent studies by Soto et al. (2005) and Olivers et al. (2006).
In these studies, participants were asked to search for a target object while actively maintaining a color or shape in VWM. On some trials, a distractor object that matched the perceptual features being maintained in VWM was present in the search array. The presence of a distractor matching VWM led to longer search times, despite the fact that the object matching memory was never the target. These data suggest that, at least under some circumstances, attention is automatically directed to items matching the content of VWM. In addition, both studies found that objects matching memory were more likely to attract eye movements, indicating VWM guidance over time scales characteristic of overt behavior. The evidence of VWM guidance in these studies is quite strong, because the content of VWM was either irrelevant to or inconsistent with the demands of the search task. In real-world search, the task ("find the pen") and the content of VWM (perceptual features of the pen retrieved from LTM) typically correspond.

It is important to note that VWM can also be used to avoid attending to items matching memory (Downing \& Dodds, 2004; Woodman \& Luck, 2007), suggesting that there is flexibility in the use of VWM to guide attention. For the search operations that occur when a saccade fails to land on the intended target, however, it is almost always the case that efficient search depends on orienting attention toward an item held in VWM, because the saccade target object is maintained prominently in VWM across the saccade, and the goal after an errant saccade is to direct attention toward that target object. In the present study, we focused on the use of VWM to guide attention toward items matching memory.

Support for the specific claim that VWM enables efficient reacquisition of a saccade target after an errant saccade comes from a recent study by Hollingworth et al. (2008). In this study, participants fixated the center of a circular array of colored disks (similar to that shown in Figure 1; the online version of this figure shows colors rather than black-and-white patterns). One disk was cued by rapid expansion and contraction, and the participant generated a saccade to that object. During the saccade

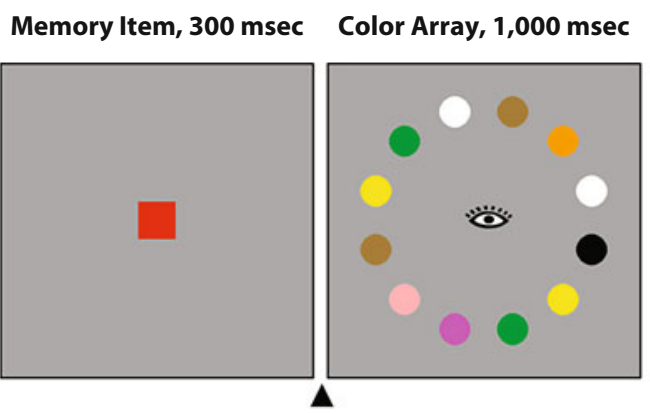

400-msec Interstimulus Interval
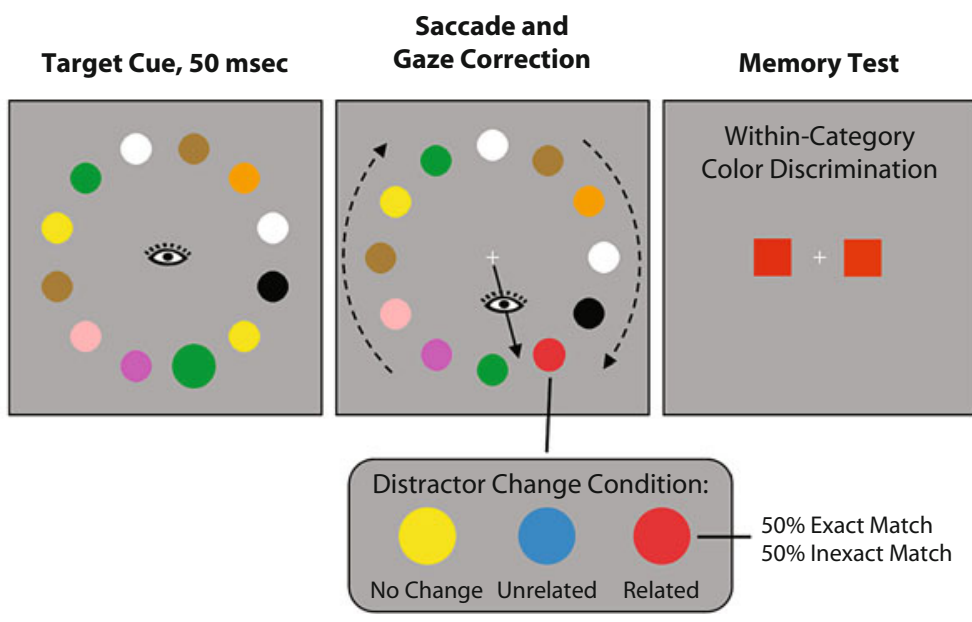

Figure 1. Sequence of events in a trial of Experiment 1. (In the printed version of this figure, the colors of the objects are represented by different fill patterns. The online version is in color.) 
(when vision is suppressed), the entire array was rotated by one half of the distance between adjacent objects, such that the position of a given object prior to the rotation was halfway between two objects after the rotation. This typically caused the eyes to land between the target object and a distractor object that was adjacent to the target (henceforward termed the distractor object), creating target ambiguity. Color was the only feature to distinguish the target and distractor objects, and the direction of array rotation could not be perceived during the initial saccade. Thus, to direct gaze to the target and not the distractor, perceptual information from before the saccade (such as the target's color) must be retained across the saccade in VWM and then compared with objects near the landing position. ${ }^{3}$

Hollingworth et al. (2008) found that VWM-based gaze correction in this paradigm was highly accurate and efficient. Participants redirected gaze to the appropriate object on essentially all trials. In addition, the use of VWM to redirect gaze to the target added only $40 \mathrm{msec}$ to the latency of the corrective saccade (compared with a singleobject control condition in which memory was not needed to correct gaze). ${ }^{4}$ Moreover, the accuracy and speed of gaze correction was impaired by a concurrent VWM load but not by a concurrent verbal WM load, demonstrating that VWM is indeed functional in search after an errant saccade, directing attention and the eyes to the original saccade target. Finally, VWM-based corrective saccades were generated even when participants were instructed to avoid making them, suggesting that correction is largely an automatized skill. This last result is broadly consistent with the involuntary orienting of attention to items matching VWM observed by Soto et al. (2005) and by Olivers et al. (2006) in more traditional search tasks.

\section{The Present Study}

An advantage of the experimental paradigm developed by Hollingworth et al. (2008) is that it assesses a relatively automatic aspect of visual search. That is, when the eyes fail to land on the intended target, participants are often unable to suppress making a second, corrective saccade to the target, and they are generally unaware that they have done so. In contrast, traditional visual search tasks depend to a large extent on voluntary strategies to establish the relevant search parameters before the search is initiated. Indeed, whereas several studies using traditional search tasks have found that attention is directed toward items that match the contents of VWM (Olivers et al., 2006; Soto et al., 2005; Soto \& Humphreys, 2008), others have found that attention can be strategically directed away from these items (Downing \& Dodds, 2004; Woodman \& Luck, 2007). By examining the relatively automatic and unconscious search process involved in reacquiring a target object after an errant saccade, one can examine the interaction among VWM, attention, and gaze control in a manner that minimizes strategic factors. In addition, the present study has the potential to illuminate the role of VWM in one of the most common types of visual search (i.e., the search that occurs following every errant saccade); presumably, this will be informative about the general relationships among VWM, attention, and gaze control.
More specifically, the present study examined whether a biased competition mechanism (Chelazzi et al., 1998; Chelazzi et al., 1993; Desimone \& Duncan, 1995) can account for the relatively automatic search operations that occur after an errant saccade. A biased competition mechanism would appear particularly well suited to explain the type of search that occurs in this situation. The shift of attention to the target prior to the saccade ensures that perceptual features of that object will be actively maintained in VWM when the eyes land. If the eyes miss the saccade target and multiple objects are competing for attention, the saccade target object will (1) match the contents of VWM, (2) be at a competitive advantage relative to other objects, (3) drive the spatial locus of attention toward itself, and (4) trigger a corrective saccade. The automatic nature of VWM-based gaze correction (Hollingworth et al., 2008) makes gaze correction precisely the sort of real-world search that would be governed by an automatic attentional bias toward objects matching memory.

To test whether VWM modulates the competition among objects during search for the original saccade target, we modified the Hollingworth et al. (2008) task to manipulate the relationship between VWM and the objects lying near the landing position of the saccade. The basic paradigm is illustrated in Figure 1. Participants completed a saccade task that was bracketed by a color memory task. In the saccade task, participants fixated the center of a circular array of 12 color disks and executed a saccade to an object that was cued by rapid expansion and contraction. Participants were instructed simply to fixate the cued object as quickly as possible. On a subset of trials, the array was rotated (either clockwise or counterclockwise) one half of the distance between adjacent objects, so that the eyes landed between the target and a distractor object, and a secondary saccade was required to foveate the target. We artificially induced saccade errors so that the landing position of the initial saccade was, on average, midway between the target and distractor. This eliminated the possibility that differences in the distance of the landing position from the target and distractor could influence performance. ${ }^{5}$ The correction of induced saccade errors is just as accurate and as rapid as the correction of naturally occurring saccade errors (Hollingworth et al., 2008).

Although the colors of the items in the circular array of color disks were not explicitly task relevant, memory for these colors was necessary for the participants to reacquire the original saccade target when gaze landed midway between the target and the distractor. Participants could not reacquire the target on the basis of perception of the rotation of the array, because the rotation was completed during the period of saccadic suppression and was not directly visible. ${ }^{6}$ In addition, clockwise and counterclockwise rotations produced exactly the same spatial arrangement of occupied locations after the saccade, so gaze could not be corrected on the basis of spatial memory. Thus, the only means to reacquire the original saccade target, and to direct the eyes to it, was to remember visual properties of the array from before the saccade (such as the target color) and compare this memory with the perceptual input after the eyes landed. 
The color memory task that bracketed the saccade task required participants to remember a single color square (see Figure 1), which was presented at fixation for $300 \mathrm{msec}$ at the beginning of the trial. Its color was drawn randomly from a set of 12 colors: 4 variants each of red, blue, and green. The saccade task was performed while this color was maintained in memory. After the completion of the saccade task, participants saw two color patches: one identical to the initial memory color and the other selected randomly from the remaining three colors in that category. Participants indicated which of the two colors matched the original color being held in memory. Thus, participants were required to retain fairly precise color information to perform the within-category discrimination, which minimized the ability to use nonvisual (e.g., verbal) representations to perform the memory task (Olivers et al., 2006).

The key manipulation was to change the color of the distractor object adjacent to the saccade target, so that the distractor either did or did not match the color being retained for the color memory task. The distractor color was changed during the initial saccade to the target object, simultaneously with the array rotation. By manipulating the color of the distractor when the eyes landed, we could determine whether a distractor that matched the features of an object in VWM would attract attention and impair the execution of a secondary saccade to the target. The three main conditions are illustrated in Figure 1.

In the no-change condition, the distractor object did not change during the initial saccade and did not match the color of the memory square. This provided a baseline measure of performance in the standard task.

In the related condition, the distractor object changed during the initial saccade to a color from the same category as the color maintained for the memory task. There were two subconditions: exact match, in which the distractor was changed so it matched exactly the color square in the memory task; and inexact match, in which the distractor was changed so that it matched the color that would be used as the foil color in the subsequent twoalternative forced choice (2AFC) test (and was therefore similar in color to the color square in the memory task). The inclusion of these subconditions discouraged strategic sampling of the distractor color, because the color of the distractor was not correlated with the correct color in the 2AFC test (Olivers et al., 2006). If the matching color had always been an exact match, the participants may have intentionally attended to this item to help them retain the color of the original memory item. The high probability of inexact matches used in the present experiment would greatly reduce the usefulness of this strategy. ${ }^{7}$ In addition, the exact and inexact match conditions allowed us to test the effect of slight color variations on the strength of distractor competition in gaze correction.

Note that, when an array disk matching the color category of the memory square was present, the matching disk was never the original saccade target and was never the object to which gaze should be corrected. Thus, participants had no incentive to attend to and fixate an object matching the color of the memory square but had signifi- cant incentive to avoid attending to such objects. This created a search task similar to previous studies in which a distractor matching memory was never the target (Olivers et al., 2006; Soto et al., 2005). If a matching distractor attracted attention in the present study, we can infer that the attraction was automatic, in the sense of not being under strategic control.

Finally, in the unrelated condition, the distractor was changed to one of the eight colors from the two color categories that were not the color category of the initial square. The related and unrelated conditions were equated for the introduction of a distractor change and for the introduction of a new color to the array. Any difference between the related and unrelated conditions could then be attributed to the factor of interest: the match between the distractor color and the color of the memory square maintained in VWM.

We hypothesized that, if VWM interacts with the sensory representation of objects that match memorybiasing selection toward matching objects - a distractor that matches an item in VWM should produce stronger competition with the saccade target than a distractor that does not match an item in VWM. That is, when the eyes land halfway between the target and distractor, the distractor should be more likely to attract attention if it matches the features of an item in VWM. This may slow the execution of a secondary saccade to the target (gaze correction latency), and it may increase the probability that gaze is directed to the distractor and therefore decrease the probability that gaze is directed to the target (gaze correction accuracy). It is important to note that we did not expect a distractor that matched memory to win the competition between target and distractor on a large proportion of trials. The saccade target object should be retained robustly in VWM across the saccade, particularly because the target is attended immediately before the saccade itself. However, a reduction in the accuracy and/or efficiency of correction to the target would indicate that the content of VWM modulated the competition among potential saccade targets. This would support the hypothesis that a biased competition mechanism provides top-down control over visual search after an errant saccade.

\section{EXPERIMENT 1}

\section{Method}

Participants. Participants in all experiments were drawn from the University of Iowa community and were between the ages of 18 and 30 . They received course credit or were paid. All reported normal or corrected-to-normal vision. Sixteen participants completed Experiment 1.

Stimuli. The background for all stimulus images was a midlevel gray. A to-be-remembered color square was presented at the beginning of the trial (Figure 1), subtending $3.3^{\circ} \times 3.3^{\circ}$. The category of that color was chosen randomly from a set three (red, green, and blue). Within the selected category, the particular value of the memory color was chosen randomly from a set of four similar colors, which were spaced approximately evenly around the color wheel. For example, in the red category, the four reds were chosen as approximately evenly spaced along the continuum of hues from red-orange to red-purple. The $x, y$, and luminance values for each color were measured with a Tektronix model J17 colorimeter (Richardson, TX) 
using the $1931 \mathrm{CIE}$ color coordinate system. The four reds were $x=$ $.53, y=.27,19.0 \mathrm{~cd} / \mathrm{m}^{2} ; x=.61, y=.30,17.8 \mathrm{~cd} / \mathrm{m}^{2} ; x=.65, y=$ $.33,17.7 \mathrm{~cd} / \mathrm{m}^{2} ;$ and $x=.63, y=.34,19.1 \mathrm{~cd} / \mathrm{m}^{2}$. The four blues were $x=.17, y=.15,11.8 \mathrm{~cd} / \mathrm{m}^{2} ; x=.16, y=.14,10.0 \mathrm{~cd} / \mathrm{m}^{2} ; x=.17$, $y=.12,9.3 \mathrm{~cd} / \mathrm{m}^{2} ;$ and $x=.17, y=.10,8.8 \mathrm{~cd} / \mathrm{m}^{2}$. The four greens were $x=.32, y=.59,33.1 \mathrm{~cd} / \mathrm{m}^{2} ; x=.30, y=.60,32.2 \mathrm{~cd} / \mathrm{m}^{2} ; x=$ $.29, y=.53,33.1 \mathrm{~cd} / \mathrm{m}^{2}$; and $x=.26, y=.44,34.5 \mathrm{~cd} / \mathrm{m}^{2}$. In the same manner, we chose a second color from one of the two remaining categories for use as the distractor color in the unrelated condition.

The circular array that was presented before the primary saccade to the target consisted of 12 color disks (Figure 1) with a central, black fixation cross. Two initial array configurations were possible, one with the objects at each of the 12 clock positions and another rotated by $15^{\circ}$. The color of each disk was chosen randomly from a set of $11\left[\mathrm{red}\left(x=.65, y=.33,16.9 \mathrm{~cd} / \mathrm{m}^{2}\right)\right.$, blue $(x=.15, y=$ $\left..08,10.4 \mathrm{~cd} / \mathrm{m}^{2}\right)$, green $\left(x=.31, y=.60,10.5 \mathrm{~cd} / \mathrm{m}^{2}\right)$, yellow $(x=$ $\left..43, y=.51,80.3 \mathrm{~cd} / \mathrm{m}^{2}\right)$, magenta $\left(x=.30, y=.15,29.0 \mathrm{~cd} / \mathrm{m}^{2}\right)$, black $\left(<.001 \mathrm{~cd} / \mathrm{m}^{2}\right)$, white $\left(81.6 \mathrm{~cd} / \mathrm{m}^{2}\right)$, brown $(x=.46, y=.42$, $\left.10.1 \mathrm{~cd} / \mathrm{m}^{2}\right)$, pink $\left(x=.41, y=.31,36.3 \mathrm{~cd} / \mathrm{m}^{2}\right)$, orange $(x=.56$, $\left.y=.40,27.9 \mathrm{~cd} / \mathrm{m}^{2}\right)$, and aqua $\left.\left(x=.22, y=.31,72.0 \mathrm{~cd} / \mathrm{m}^{2}\right)\right]$, with two constraints. First, color repetitions had to be separated by at least two objects. Second, none of the colors could match (1) the category from which the memory square color was chosen or (2) the category from which the distractor color was chosen for the unrelated condition. (Although this latter color was used only in the unrelated condition, it was eliminated from inclusion in the array in all conditions to equate the composition of the array.) Consider the sample trial in Figure 1. In this example, the memory square (and hence the related distractor color) came from the red category, and the unrelated distractor color came from the blue category. Thus, the presaccade array of disks could not include either red or blue. The exclusion of red and blue ensured that, in the presaccade array, none of the color disks matched what would become the distractor color category in the related condition (red) or in the unrelated condition (blue). This also ensured that none of the colors in the presaccade array matched the color retained in VWM from the color memory task, preventing a color match from influencing the primary saccade and limiting the potential effect of a color match to the secondary saccade.

Color disks subtended $1.6^{\circ}$ and were centered $5.9^{\circ}$ from central fixation. The distance between the centers of adjacent disks was $3.0^{\circ}$. The saccade target was equally likely to appear at each of the 12 possible locations. When the target was cued, it expanded to $140 \%$ of its original size and contracted back to the original size over $50 \mathrm{msec}$ of animation. The angular difference between adjacent disks was $30^{\circ}$. For rotation trials, the array was rotated $15^{\circ}$ clockwise on half the trials and $15^{\circ}$ counterclockwise on the other half.

When the array rotated during the saccade to the target, the distractor either retained its original color (no change), was changed to match the color category of the remembered square (related), or was changed to the color drawn from one of the two nonremembered color categories (unrelated). In the related condition, the distractor changed either to the exact color of the remembered square (exact match) or to one of the remaining three colors within that category selected randomly (inexact match). In the latter case, the inexact match color would become the foil color in the 2AFC memory test following gaze correction. Note that the relevant distractor object (i.e., the one that changed color in the related and unrelated conditions) depended on the direction of array rotation. When the array rotated clockwise, the distractor object was the object adjacent to the target in the counterclockwise direction (see Figure 1). This assignment was reversed for counterclockwise rotations.

In the color test display, two $1.6^{\circ} \times 1.6^{\circ}$ color squares were presented, one on each side of the central fixation point, centered $2.5^{\circ}$ from that point. One color was identical to the memory square presented at the beginning of the trial (target). The other was drawn randomly from the remaining three colors in that category (foil). The positions of the two colors were determined randomly. The withincategory discrimination minimized the role of verbal encoding in the task. This method was used instead of a standard articulatory suppression task, because overt articulation produces head movements that would have impaired precise eyetracking.

Stimuli were displayed on a $17-$ in. CRT monitor with a $120-\mathrm{Hz}$ refresh rate. Eye position was monitored by a video-based, SR Research EyeLink 2000 eyetracker sampling at $1000 \mathrm{~Hz}$. The right eye was tracked. A chin and forehead rest was used to maintain a constant viewing distance of $70 \mathrm{~cm}$ and to minimize head movement. A computer running E-Prime software controlled the experiment. Gaze position samples were streamed in real time from the eyetracker to the computer running E-Prime. E-Prime then used gaze position data to control trial events (such as transsaccadic rotation). Manual responses to the color test were collected by a serial button box interfaced with the E-Prime software.

Procedure. The experimenter initiated each trial after eyetracker calibration was checked. Following a 350-msec delay, the color memory square was presented for $300 \mathrm{msec}$. There was a $400-\mathrm{msec}$ blank (fixation cross only) interstimulus interval. Then, the color disk array was presented for $1,000 \mathrm{msec}$ while participants maintained central fixation. Next, the target cue animation was presented for $50 \mathrm{msec}$. Participants were instructed to generate an eye movement to the target as quickly as possible. They were informed about the possibility of array rotations and distractor changes but were told that the task under these circumstances remained the same: to fixate the original target object as quickly as possible.

The array was rotated during the primary saccade on $55 \%$ of trials, typically causing the eyes to land between the target and an adjacent distractor. No-rotation trials were included so that participants could not reliably predict whether there would be an array rotation. A rotation was accomplished by replacing the original array (within a single refresh cycle) with a new array that was rotated $15^{\circ}$ clockwise or counterclockwise. On related and unrelated trials, the rotated array contained a distractor color change. After the eyes landed and the original target had been fixated, the target was outlined by a box for $400 \mathrm{msec}$ to indicate successful completion of the saccade task. Next, the color test display was presented until manual response. Participants pressed the left- or right-hand button to indicate whether the object on the left or right, respectively, matched the original memory square. An incorrect response was followed by presentation of the word "incorrect" for $1 \mathrm{sec}$.

Array rotation during the primary saccade to the target was accomplished using a boundary technique. Participants initially fixated the center of the array. An invisible, circular boundary was defined with a radius of $1.3^{\circ}$ from central fixation. After the cue, E-Prime monitored for an eye position sample beyond the circular boundary, and, on array rotation trials, the rotated array was then written to the screen (on no-rotation trials, a new image was also written to the screen during the saccade, but it was the same as the preview image). Pilot testing ensured that screen changes were completed before the beginning of the next fixation. The direction of rotation could not be perceived during the saccade, because of visual suppression during the saccade and masking generated by the postsaccade perceptual input (for a review, see Matin, 1974).

After receiving instructions, participants completed 12 practice trials, drawn randomly from the full design. This was followed by an experiment session of 264 trials: 120 no-rotation trials and 144 rotation trials. Rotation trials were divided evenly among the three distractor change conditions: no change, unrelated, and related. Trial order was determined randomly. The entire session lasted approximately $1 \mathrm{~h}$.

Data analysis. Dedicated software was used to analyze eyetracking data offline. A velocity criterion (eye rotation $>30^{\circ} / \mathrm{sec}$ ) was used to define saccades. These data were analyzed with respect to critical regions in the image, such as the target and distractor regions, allowing us to determine whether the eyes were directed first to the target region or to the distractor region and the latency of any such correction. Object scoring regions were circular and had a diameter of $1.9^{\circ}, 20 \%$ larger than the color disks themselves. 
Rotation trials were eliminated from analysis if the primary saccade landed on an object rather than between the target and adjacent distractor, if more than one saccade was required to bring the eyes from central fixation to the general region of the object array, or if the eyetracker lost track of the eye. The majority of eliminated trials were those in which the primary saccade landed on an object, reflecting the fact that saccades are often inaccurate. A total of $17 \%$ of the rotation trials were eliminated in Experiment 1.

The mean latency of the primary saccade to the target (timed from the beginning of the cue animation) was $244 \mathrm{msec}$. As expected, the average landing position of the primary saccade on rotation trials was midway between the target and distractor. Mean landing position was $1.83^{\circ}$ from the center of the target and $1.84^{\circ}$ from the center of the distractor. Because the eyes tended to undershoot the array slightly, these distances were a little larger than half of the distance between the target and distractor $\left(1.5^{\circ}\right)$.

\section{Results}

The rotation trials were of central interest for examining the effect of VWM on the competition between target and distractor during search for the original saccade target. Figure 2 shows the key results for each of the three distractor change conditions for rotation trials. The top graph reports mean gaze correction accuracy, the middle graph mean correction latency, and the bottom graph mean accuracy on the color memory test. For the gaze correction data analyses, there was no effect of exact versus inexact match (see insets in Figure 2), and the data from these two subconditions were combined in the statistical analyses.

Gaze correction accuracy. Gaze correction accuracy is the proportion of trials on which the eyes were directed first to the target object after the eyes landed between target and distractor. Gaze correction accuracy was nearly perfect when the distractor remained constant in the nochange condition, consistent with our previous study of memory-dependent gaze correction (Hollingworth et al., 2008). Accuracy was somewhat lower when the distractor changed to a color that was unrelated to the color being held in memory, and it was reduced further when the distractor color was similar to or identical with the item being held in memory. This pattern led to a significant overall effect of distractor condition on correction accu$\operatorname{racy}[F(2,30)=10.8, p<.001]$. Planned follow-up comparisons demonstrated that accuracy was reliably lower in the unrelated condition than in the no-change condition $[F(1,15)=6.3, p=.024]$ and was also lower in the related condition than in the unrelated condition $[F(1,15)=5.61$, $p=.032]$.

Gaze correction latency. Gaze correction latency is the duration of the fixation before the corrective saccade when only one corrective saccade is required to fixate the target (i.e., on "correct" gaze corrections). Outlier latencies above $500 \mathrm{msec}(4.3 \%$ of the data) were eliminated from the analysis, which did not influence the pattern of results in any experiment. The overall mean correction latency was just above $200 \mathrm{msec}$, showing the remarkable efficiency with which VWM guides the eyes to the saccade target. That is, within a little more than $200 \mathrm{msec}$, on average, memory was used to select the appropriate object from among at least two alternatives and a secondary saccade was computed and initiated.
Just as gaze correction accuracy was reduced when the distractor matched the item being held in memory, gaze correction latency increased on those trials. There was a reliable overall effect of distractor condition on correction latency $[F(2,30)=3.56, p=.041]$. Correction latencies in the unrelated and no-change conditions were nearly identical $[F(1,15)=1.41, p=.25]$. There was a nonsignificant trend toward higher latency in the related condition than in the unrelated condition $[F(1,15)=$ $2.84, p=.112]$. Finally, latency was significantly higher in the related condition than in the no-change condition $[F(1,15)=4.75, p=.046]$. Thus, when the distractor matched the color of the item being held in VWM, both the accuracy and the speed of gaze correction to the target were impaired.

Color memory accuracy. There was no significant overall effect of distractor condition on color memory accuracy $[F(2,30)=1.80, p=.182]$. However, there was an effect of exact versus inexact match in the related condition $[F(1,15)=4.64, p=.048]$ (see inset graph in the bottom panel of Figure 2). Accuracy was higher when the distractor was an exact match for the remembered color than when it was an inexact match. Color memory performance on no-rotation trials was $75.0 \%$ correct, which was almost identical to performance on rotation trials without a change in distractor color $(74.1 \%$ correct $)$.

\section{Discussion}

In Experiment 1, the presence of a distractor that matched the color of an object in VWM produced significant interference with the participant's ability to fixate the target following an errant saccade. This interference was observed primarily as a greater probability that the secondary, corrective saccade was directed to the distractor rather than to the target. There was a complementary but small effect on the latency of the correction. These data indicate that VWM modulates the competition between target and distractor object and that search for the saccade target is therefore guided by the relationship between the content of VWM and the perceptual properties of objects near the landing position of the saccade.

In addition to this main finding, the presence of a distractor change alone, even when the distractor did not match memory, produced a small but significant reduction in gaze correction accuracy. In order for this to occur, the color of the distractor object must have been encoded and maintained across the saccade, suggesting that transsaccadic VWM is not limited to the target object. This is consistent with research showing that VWM can store approximately three colors (Luck \& Vogel, 1997; Zhang \& Luck, 2008). However, these data should be treated with considerable caution. The effect was quite small and was not replicated in Experiments 2 and 3.

Finally, in the related condition, there was no effect of exact versus inexact match on gaze correction. This is not particularly surprising, given that the color differences within a category were very small compared with the color differences across categories. However, there was an effect of exact versus inexact match on the color 

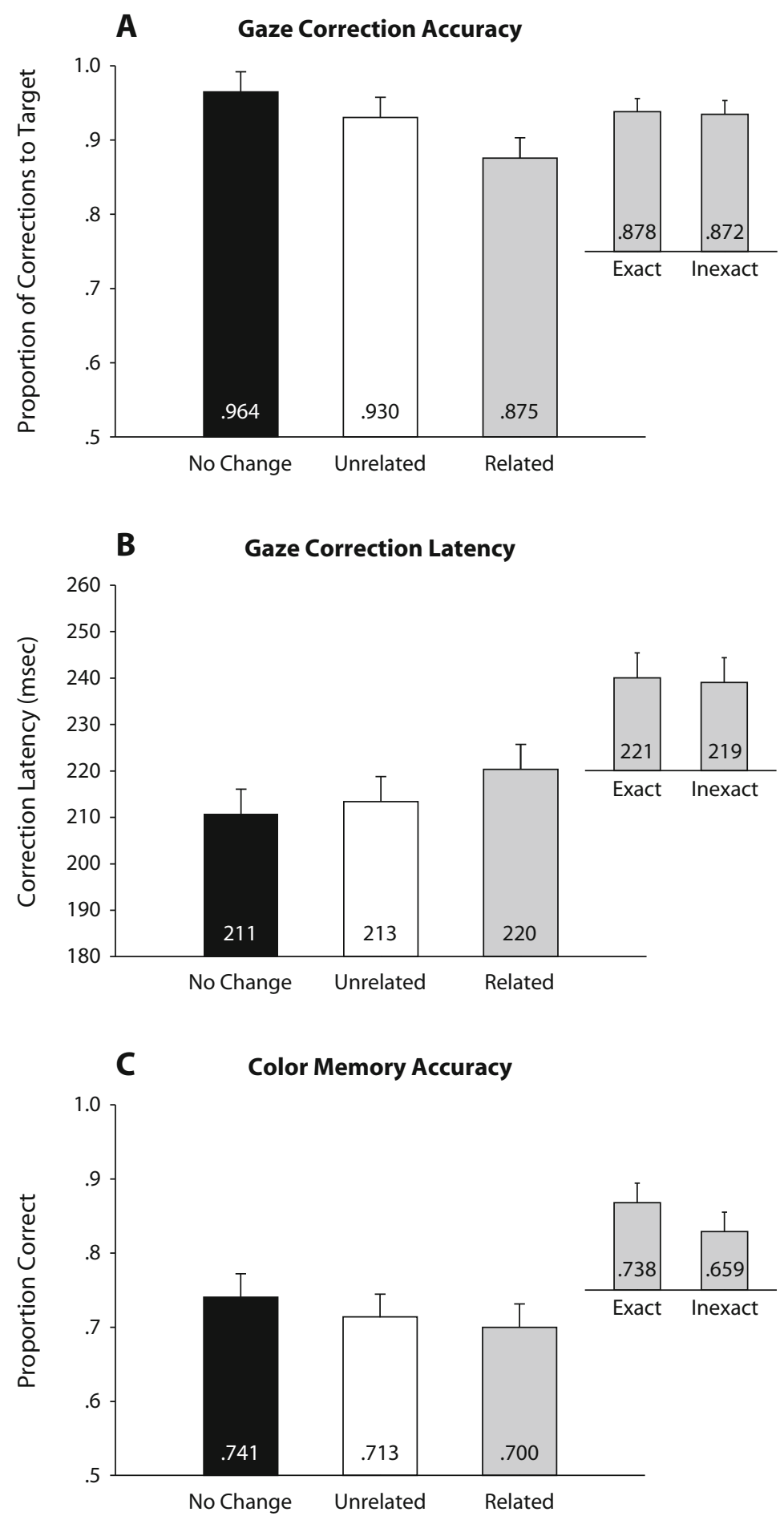

Figure 2. Experiment 1 data. (A) Gaze correction accuracy. (B) Gaze correction latency. (C) Color memory accuracy. The inset bars show performance on the exact match and inexact match trials of the related condition. Error bars are $95 \%$ confidence intervals based on the error term of the distractor change effect. 


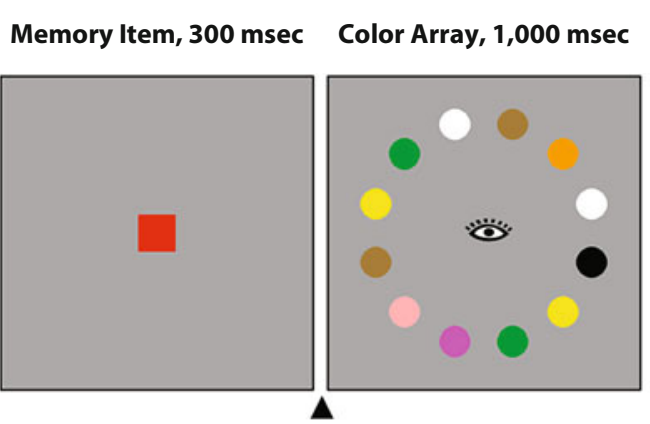

400-msec Interstimulus Interval

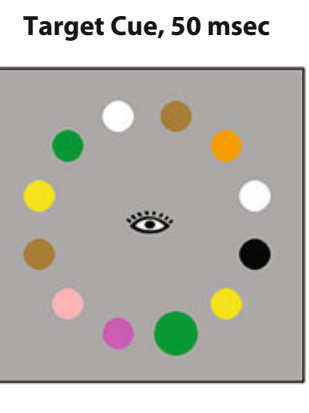

Saccade and

Gaze Correction

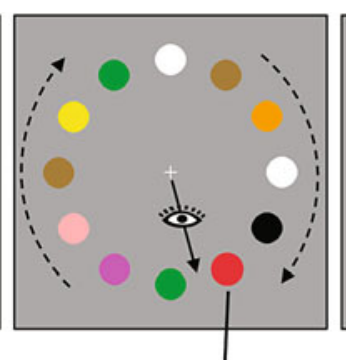

Memory Test

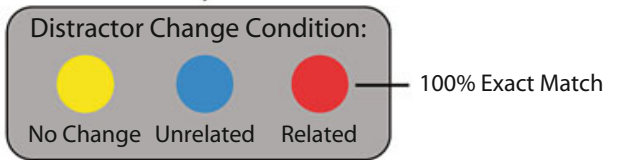

Figure 3. Sequence of events in a trial of Experiment 2. (In the printed version of this figure, the colors of the objects are represented by different fill patterns. The online version is in color.)

memory task. Color memory performance was higher when the distractor was an exact match than when it was an inexact match. A plausible explanation for this effect is that on some proportion of trials, attention was directed to the distractor and information from the distractor was consolidated into VWM. When the distractor was an exact match, consolidation of the distractor in VWM facilitated color memory performance. However, when the distractor was an inexact match (i.e., the color that would be the foil on the 2AFC test), memory performance was impaired. It is unlikely that such sampling of the distractor was strategic, because the color of the distractor was uncorrelated with the correct response on the 2AFC test. However, it is at least possible that participants thought that strategically attending to the distractor would improve memory performance, which might account for the secondary saccades directed to the distractor. To ensure that the principal gaze correction effects were not caused by strategic sampling of the related distractor, color was made irrelevant to the memory task in Experiment 2.

\section{EXPERIMENT 2}

The memory task was changed from a color discrimination task to a size discrimination task (see Figure 3; the online version of this figure shows colors rather than blackand-white patterns). A memory square was presented at the beginning of the trial. At the end of the trial, two squares were presented that differed in size, and the participant reported which of the two matched the size of the memory square. Color was an incidental property of the squares. The color of the memory square varied from trial to trial, but, within a trial, the memory square and the two test squares had exactly the same color. As in Experiment 1, in the related condition, the distractor changed to match the color of the object maintained in the memory task. This was always an exact match. In all other respects, the design of Experiment 2 was the same as in Experiment 1.

\section{Method}

Participants. Sixteen new participants completed the experiment

Stimuli and Procedure. The stimuli for Experiment 2 were identical to those in Experiment 1, with the following exceptions. The width/height of the initial memory square was chosen randomly within a range between $1.3^{\circ}$ and $2.6^{\circ}$. In the memory test image, two squares were presented, one $3.3^{\circ}$ to the left and one $3.3^{\circ}$ to the right of central fixation. One test square was the same size as the initial memory square. The other was randomly chosen to be $0.3^{\circ}$ larger or smaller than the initial memory square. Participants pressed the left or right button to indicate which of the two squares matched the size of the memory square. The color of the two test squares was identical to the color of the initial memory square. Finally, in the related condition, the changed distractor was always an exact match for the color of the memory square.

Data analysis. A total of $18 \%$ of the rotation trials were eliminated from the analysis for the reasons discussed in Experiment 1.

The mean latency of the initial saccade to the target was $242 \mathrm{msec}$. The average landing position of the initial saccade on rotation trials was midway between the target and distractor. Mean landing position was $1.93^{\circ}$ from the center of the target and $1.92^{\circ}$ from the center of the distractor.

\section{Results}

Figure 4 shows the key results for each of the three distractor change conditions.

Gaze correction accuracy. Although participants had no motivation to remember the color of the memory item or to use it in the saccade task, we found that gaze correction accuracy was substantially lower when the distractor item changed to the same color as the memory item compared with when it changed to a different color or did not change. This led to a significant overall effect of distractor condition $[F(2,30)=10.0, p<.001]$. Accuracy was slightly lower in the unrelated condition than in the no-change condition, but this effect was not significant $[F(1,15)=2.91, p=.109]$. However, accuracy was reliably lower in the related condition than in the unrelated condition $[F(1,15)=8.22, p=.012]$.

Gaze correction latency. Outlier latencies above $500 \mathrm{msec}$ were eliminated from the analysis $(3.8 \%$ of the 


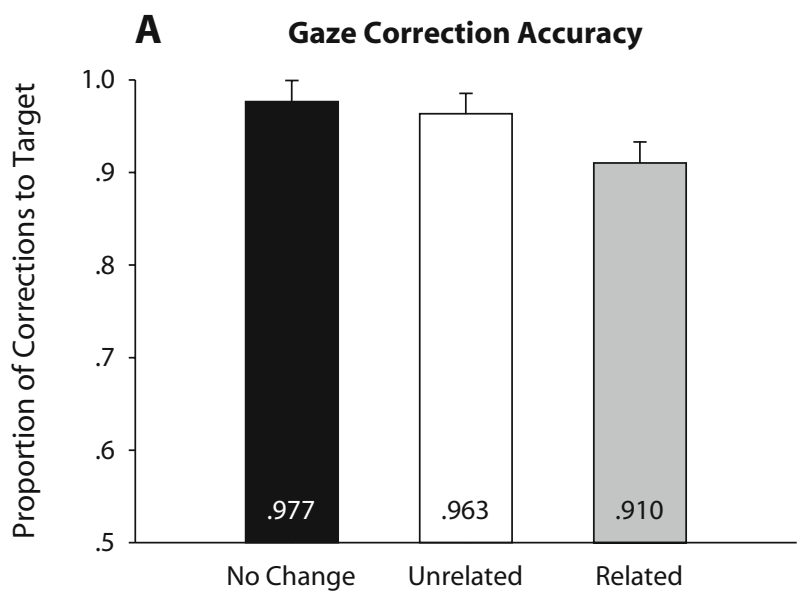

B Gaze Correction Latency
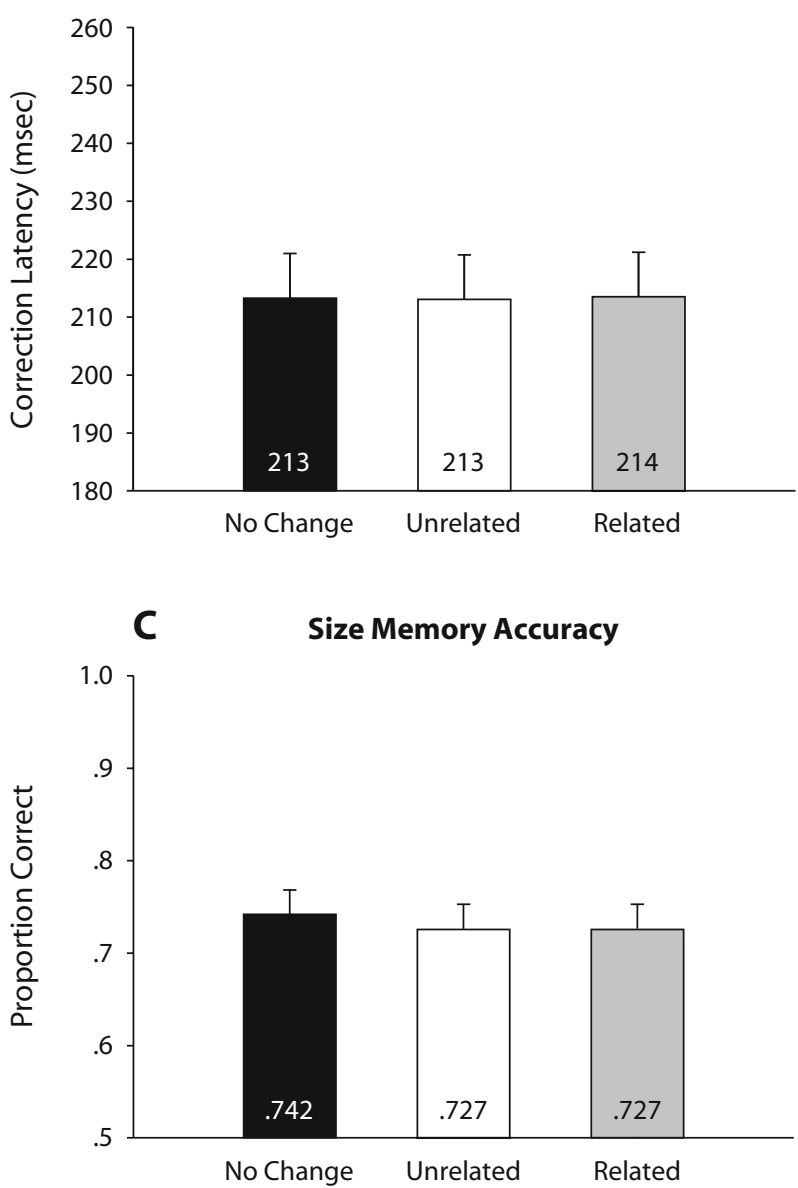

Figure 4. Experiment 2 data. (A) Gaze correction accuracy. (B) Gaze correction latency. (C) Size memory accuracy. Error bars are $95 \%$ confidence intervals based on the error term of the distractor change effect.

data). There was no effect of distractor condition on correction latency $(F<1)$.

Size memory accuracy. There was no effect of distractor condition on memory accuracy $(F<1)$. Size discrimination performance on no-rotation trials was $74.8 \%$ correct, which was nearly identical to performance on rotation trials without a change $(74.2 \%$ correct).

\section{Discussion}

Despite the fact that color was incidental to the memory task, a distractor matching the color of the memory object produced significant interference with the process of fixating the target following an errant saccade. This interference was observed as a higher probability that the secondary saccade was directed to the distractor when it matched the content of memory than when it did not. Participants could not have construed any advantage to strategically sampling the related distractor, because the color of the distractor could not plausibly have had any relevance to the later size discrimination test. Thus, incidental properties of objects appear to be maintained in VWM and can influence the competition among objects during search for the saccade target, which is consistent with the general claim that VWM maintains integrated object representations (Luck \& Vogel, 1997).

\section{EXPERIMENT 3}

In Experiments 1 and 2, we have assumed that it is the active maintenance of the initial color square in VWM that generates interference with the secondary saccade to the target. To demonstrate that active memory is necessary, in Experiment 3 we eliminated the requirement that participants remember the color square, which should eliminate any effects of the match between this square and the distractor in the saccade task (Olivers et al., 2006; Soto et al., 2005). Participants viewed the color square at the beginning of the trial, but there was no test of memory at the end of the trial. If it is the active maintenance of object properties in VWM that guides attention to matching items, we should not observe gaze correction interference in this experiment.

\section{Method}

Participants. Sixteen new participants completed the experiment.

Stimuli and Procedure. The stimuli and procedure for Experiment 3 were identical to those in Experiment 1, except there was no color test at the end of the trial. In addition, in the related condition, the changed distractor color was always an exact match of the initial color square. To provide a plausible reason for the appearance of the color square at the beginning of the trial, participants were instructed that the appearance of the color square signaled that the trial was about to begin.

Data analysis. A total of $18 \%$ of the rotation trials were eliminated from the analysis for the reasons discussed in Experiment 1.

The mean latency of the initial saccade to the target was $230 \mathrm{msec}$. The average landing position of the primary saccade on rotation trials was midway between the target and distractor. Mean landing position was $1.75^{\circ}$ from the center of the target and $1.73^{\circ}$ from the center of the distractor.

\section{Results}

Figure 5 shows the key results for each of the three distractor change conditions.

Gaze correction accuracy. There was no effect of distractor condition on correction accuracy $(F<1)$. A power analysis indicated that the experiment had sufficient power to detect an accuracy difference between the related and un- 


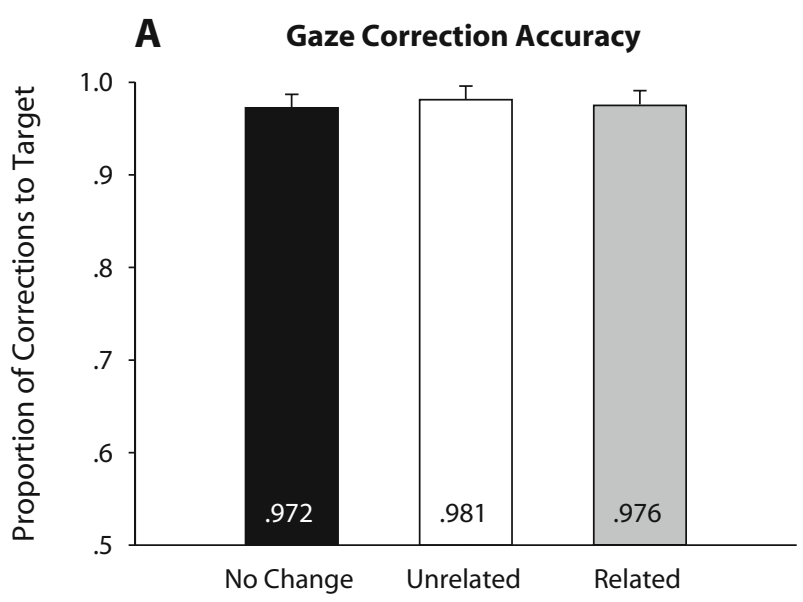

B Gaze Correction Latency

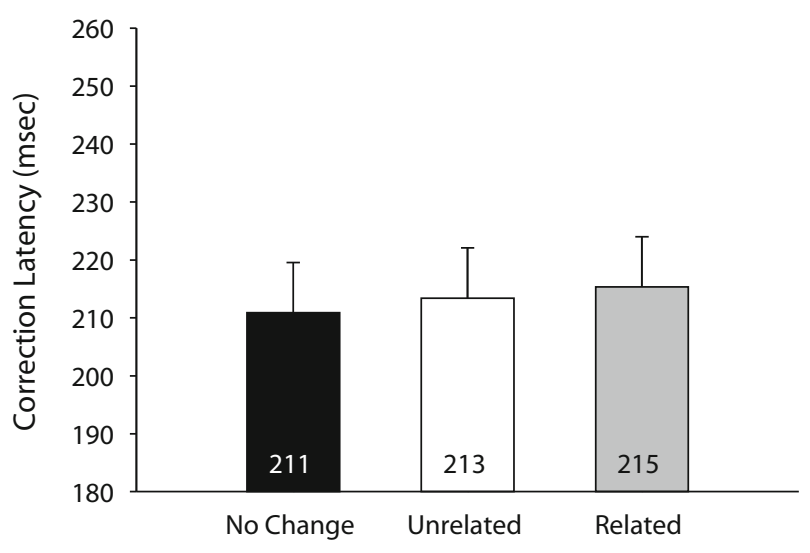

Figure 5. Experiment 3 data. (A) Gaze correction accuracy. (B) Gaze correction latency. Error bars are $\mathbf{9 5 \%}$ confidence intervals based on the error term of the distractor change effect.

related conditions of $1.5 \%$. Thus, if an effect of the magnitude observed in Experiments 1 (5.5\%) and $2(5.3 \%)$ had been present, there was sufficient power to have detected it.

Gaze correction latency. Outlier latencies above $500 \mathrm{msec}$ were eliminated from the analysis $(3.6 \%$ of the data).There was no effect of distractor condition on correction latency $(F<1)$.

\section{Discussion}

When there was no demand to remember the initial color square across the trial, there was no evidence of gaze correction interference when the distractor changed to match the initial color square. Thus, the interference observed in Experiments 1 and 2 cannot be explained by mere exposure to a matching color. Instead, interference requires active maintenance of the object color in VWM.

\section{GENERAL DISCUSSION}

Top-down control over visual search is required to ensure that the eyes are efficiently directed to task-relevant objects. One general means by which top-down control can be exerted is through interactions between VWM and attention. Perceptual features of an object are activated in VWM, and this activation then biases attention toward visible objects sharing those features (Chelazzi et al., 1993; Desimone \& Duncan, 1995; Olivers et al., 2006; Soto et al., 2005). In the present study, we examined a particular form of visual search that is critical for ensuring that the eyes arrive at an intended object. A visual search operation is performed routinely whenever a saccade fails to land on the intended target object (Hollingworth et al., 2008; Richard et al., 2008); the original target object must be reacquired, and gaze must be corrected to that object via a secondary saccade. We examined the possibility that, in this particular type of highly practiced visual search, the maintenance of object properties in VWM automatically biases attention toward objects matching those properties, supporting a rapid correction of gaze to the original saccade target.

A biased-competition mechanism would appear to be well suited to explaining search and gaze correction after an errant saccade. First, gaze correction is certainly one of the most common forms of visual search behavior; thus, it is likely to depend on general mechanisms of VWMmodulated competition observed in standard visual search tasks (Olivers et al., 2006; Soto et al., 2005). Second, a close relationship among VWM, attention, and gaze control has already been well established. Before a saccade, attention precedes the eyes to the target object, which supports the consolidation of saccade target properties into VWM. Saccade target properties encoded immediately before the saccade could then be used to guide attention to the target when the eyes fail to land on it. In fact, it is quite possible that this is one of the central reasons that attention does indeed precede the eyes to the saccade target (see Luck, 2009).

To test this hypothesis, we induced saccade errors by rotating an array of color disks during a saccade to one of them. The eyes tended to land between the target object and an equidistant distractor object, and gaze correction to the target was required. In addition, participants maintained a color square in VWM throughout the trial. On some trials, the color of the distractor object was changed during the initial saccade so that it matched the color square in VWM. A distractor that matched memory was at a competitive advantage compared with a distractor that did not match memory, generating significant interference with the process of reacquiring the target and correcting gaze to it. Interference was observed even when color was an incidental property of the memory task object. However, interference was eliminated when the need to remember the memory square was removed; VWM-modulated competition was dependent on the active maintenance of the memory object in VWM. Together, these data suggest that VWM does indeed modulate the competition between objects during search for the saccade target, leading to a competitive advantage for objects that match the content of VWM.

In the present method, we created a situation in which a second object (the memory square) was maintained in VWM along with the saccade target object, and a nearby object matched that second object. In natural vision, how- 
ever, such a circumstance would be rare. The target object will almost always be the dominant object in VWM (Currie, McConkie, Carlson-Radvansky, \& Irwin, 2000; Henderson \& Hollingworth, 1999, 2003; Irwin, 1992; Irwin \& Andrews, 1996; Irwin \& Gordon, 1998), particularly because the capacity of VWM for real-world objects is highly limited (Alvarez \& Cavanagh, 2004; Hollingworth, 2004), and the saccade target will always be the object most recently attended before gaze correction, with target object properties encoded into VWM immediately before the saccade. Thus, under normal circumstances, the competitive advantage afforded to an object matching memory will result in accurate and efficient gaze correction to the target. Indeed, gaze correction in the no-change condition, in which the only object matching memory was the saccade target, was nearly perfect ( $>96 \%$ correct) and quite rapid $(\sim 210 \mathrm{msec})$, consistent with prior demonstrations that VWM is used in a highly efficient manner to guide corrective saccades (Hollingworth et al., 2008). As we have argued before, given the almost constant need to ensure that the eyes are directed efficiently to goal-relevant objects, gaze correction is likely to be one of the central functions of the VWM system.

The present results are generally consistent with previous experiments examining the guidance of attention by VWM in more traditional search tasks. First, our results replicated the finding of automatic attentional attraction to items matching VWM (Olivers et al., 2006; Soto et al., 2005; Soto \& Humphreys, 2008; Soto et al., 2006), with significant distractor interference, even though the distractor property was never shared by the target. Second, we replicated effects of VWM on eye movements during search (Olivers et al., 2006; Soto et al., 2005), with a greater proportion of saccades directed to the distractor when it matched VWM. Furthermore, we replicated the finding that a matching distractor produces interference only when its properties are actively maintained in VWM (Olivers et al., 2006; Soto et al., 2005).

There was one key difference between the results of the present study and those using more traditional search tasks: the ability of incidental object properties to influence search performance. In Experiment 4 of Olivers et al. (2006), participants saw a colored shape at the beginning of the trial. At the end of the trial, memory was tested for shape alone or for color alone, and the type of test was blocked. Thus, participants could perform the memory task by remembering only one of the two features of the object. In the search task that was bracketed by the memory task, a distractor could be present that matched either the relevant or the irrelevant feature in the memory task. Significant interference was observed only for the relevant feature, suggesting that incidental features of the memory object were not retained in VWM or were not functional in biasing attention to a matching object.

It is not entirely clear why Olivers et al. (2006) failed to find an effect of incidental features, whereas we did. In Olivers et al., both features of the memory object were tested, but in different blocks. In the present Experiment 2, the incidental feature (color) was never tested, providing a conservative assessment of the effect of incidental features on search. However, in the present experiments, color was relevant to the saccade task (because correction required memory for color), whereas color was never relevant to the search task in Olivers et al. Thus, the color demands of our saccade task might have been generalized to the size memory task, despite the fact that color was irrelevant to that task.

More generally, the different results could have been caused by differences in the informativeness of incidental features to the two types of search task. If, in a standard visual search task, one is searching for any pen present in a scene, the color of a potential target object might not be centrally relevant to determining whether it is or is not a pen, because pens come in many different colors. However, in the realm of real-world gaze correction, incidental color could be highly informative as a means to discriminate the particular pen toward which gaze is being directed from other nearby objects after an errant saccade (Richard et al., 2008). For example, if the pen is green, that attribute might not be strongly related to the goals of the larger search task, because green is not a common color for a pen. However, if the green pen is the only green object near the landing position of an errant saccade, its remembered color could serve to discriminate it from other nearby objects, enabling efficient gaze correction. Because incidental features of an object will often be informative for gaze correction independently of their informativeness to the broader search task, it is possible that the search operations supporting gaze correction consult incidental features to a greater extent than they are consulted in more conventional search paradigms.

In the present study, we have focused on the attraction of attention to items matching VWM. There remains a debate over whether attraction toward items in VWM results from a fixed relationship between VWM and attention or whether participants can flexibly control the relationship between items in VWM and the orienting of attention. As discussed above, some studies have suggested that the attraction of attention to objects matching VWM is automatic, with the presence of a matching distractor interfering with search, even if it is never the target object (Olivers et al., 2006; Soto et al., 2005; Soto \& Humphreys, 2008). However, results of two studies using very similar experimental designs have indicated the reverse effect (Downing \& Dodds, 2004; Woodman \& Luck, 2007). The presence of a distractor matching memory led to slightly faster search in these two studies, presumably because participants knew that an object matching memory was never the target and could exclude that item from search.

The source of the discrepancy between these studies is not readily apparent. Soto and Humphreys (2008) suggested that studies failing to find automatic allocation of attention to matching items have used articulatory suppression to minimize verbal encoding, whereas studies that have observed interference from a matching distractor have not used articulatory suppression. It is worth noting that the present study did not use articulatory suppression; a within-category discrimination was used instead to minimize verbal encoding of the stimuli. However, it seems unlikely that articulatory suppression is the criti- 
cal difference between studies. Although articulatory suppression could potentially draw on general working memory resources and disrupt the use of VWM to guide attention (Soto \& Humphreys, 2008), it is unclear how articulatory suppression could explain the reversal of the effect of a matching distractor observed by Woodman and Luck (2007) and Downing and Dodds (2004). In addition, a recent direct test of articulatory suppression found that it could not account for the differences among studies (Olivers, in press).

In the context of gaze correction, the attraction of attention to items matching memory would seem to be the standard mode of operation. In real-world tasks, it is unlikely that search for the saccade target after an errant saccade would ever be set so as to avoid correcting gaze to a particular object that was being held in memory, because the saccade target object will likely be the object retained most robustly across the saccade. In addition, the brief time course of saccades and gaze correction would provide little opportunity to develop the relatively elaborate task set necessary to exclude a particular remembered item from search. Thus, it is possible that in more deliberate forms of search, the relationship between VWM and attention can be flexibly set, whereas, for more rapid forms of search, such as gaze correction, attention is oriented automatically (in the sense of not being under strategic control) to items matching VWM.

Finally, although the behavioral data presented here do not allow us to draw strong conclusions about the underlying neural mechanisms of memory-based gaze correction, the present results can be accommodated quite naturally by an extension of existing neural models of VWM, attention, and saccade target selection. Prior to a saccade, spatial attention shifts to the location of the impending eye movement, as observed in frontal eye fields (FEF; Sato \& Schall, 2003; Schall, 2002) and the superior colliculus (McPeek \& Keller, 2002). The selection of the saccade endpoint feeds back into visual sensory regions (Moore \& Armstrong, 2003), generating enhanced sensory processing of the object at the saccade target location (Moore \& Fallah, 2004; Müller, Philiastides, \& Newsome, 2005) and selective activation of a subset of neurons in the ventral stream coding the visual properties of that object (Moore \& Armstrong, 2003; Moore, Tolias, \& Schiller, 1998; Sheinberg \& Logothetis, 2001). Thus, before the saccade, the shift of attention selectively activates saccade target features. During the saccade, ventral stream activation corresponding to the visual features of the target is maintained in the absence of direct sensory input (Chelazzi et al., 1998; Chelazzi et al., 1993); that is, saccade target properties are maintained in VWM. If the saccade fails to land on the target, objects near the landing position of the saccade compete for selection as the target of the corrective saccade. This competition is modulated by the ventral preactivation of the saccade target's features, and the object matching the preactivated features typically comes to dominate the sensory response (Chelazzi et al., 1993; Desimone \& Duncan, 1995). This selective operation in extrastriate visual areas then feeds into those brain regions, such as FEF, that are responsible for selecting the target location of the corrective saccade, and a corrective saccade to the location of the original target is generated (similar to accounts of the effect of intertrial feature priming on saccade target selection; Bichot $\&$ Schall, 2002). One could conceptualize the influence of VWM on selection of the corrective saccade target as a memory-driven increase in the perceptual salience of the target object relative to other nearby objects that do not match the remembered target features.

\section{CONCLUSION}

The correction of gaze after an errant eye movement is critical for ensuring that the eyes are efficiently directed to task-relevant objects in the world, and gaze correction is certainly one of the most common forms of visual search behavior. Because saccade targets are attended immediately before the saccade, perceptual features of the target are encoded into VWM, are retained across the saccade, and can be used to find the target when the eyes fail to land on the intended object. In the present study, we demonstrated that a general account of the relationship between VWM and attention during search, the biased competition model (Chelazzi et al., 1993; Desimone \& Duncan, 1995), provides a good explanation of the means by which VWM is used to correct gaze. When the eyes land, objects near the landing position compete for selection as the target of the corrective saccade. This competition is modulated by the content of VWM, with objects matching memory placed at a competitive advantage for selection as the corrective saccade target. Because the saccade target object will typically be the object most prominently maintained in VWM when the eyes land, this mechanism provides a reliable and efficient means to ensure that saccade errors are corrected and that the eyes are directed rapidly to the intended object.

\section{AUTHOR NOTE}

The present research was supported by NIH Grant R01EY017356. We thank Ed Vul for helpful discussions of the research and Chris Olivers for comments on an earlier version of the manuscript. Correspondence concerning this article should be addressed to A. Hollingworth, University of Iowa, Department of Psychology, 11 Seashore Hall E, Iowa City, IA, 52242-1407 (e-mail: andrew-hollingworth@uiowa.edu).

\section{REFERENCES}

Alvarez, G. A., \& Cavanagh, P. (2004). The capacity of visual shortterm memory is set both by visual information load and by number of objects. Psychological Science, 15, 106-111.

BECKER, W. (1972). The control of eye movements in the saccadic system. Bibliotheca Ophthalmologica, 82, 233-243.

BECKER, W., \& JÜRGENS, R. (1979). An analysis of the saccadic system by means of double step stimuli. Vision Research, 19, 967-983.

Bichot, N. P., \& Schall, J. D. (2002). Priming in macaque frontal cortex during popout visual search: Feature-based facilitation and location-based inhibition of return. Journal of Neuroscience, 22, 4675-4685.

Chelazzi, L., Duncan, J., Miller, E. K., \& Desimone, R. (1998). Responses of neurons in inferior temporal cortex during memory-guided visual search. Journal of Neurophysiology, 80, 2918-2940.

Chelazzi, L., Miller, E. K., Duncan, J., \& Desimone, R. (1993). A neural basis for visual search in inferior temporal cortex. Nature, 363, 345-347. 
Currie, C. B., McConkie, G. W., Carlson-Radvansky, L. A., \& Irwin, D. E. (2000). The role of the saccade target object in the perception of a visually stable world. Perception \& Psychophysics, 62, 673-683.

Desimone, R., \& Duncan, J. (1995). Neural mechanisms of selective visual attention. Annual Review of Neuroscience, 18, 193-222.

Deubel, H., \& Schneider, W. X. (1996). Saccade target selection and object recognition: Evidence for a common attentional mechanism. Vision Research, 36, 1827-1837.

Deubel, H., Wolf, W., \& Hauske, G. (1982). Corrective saccades: Effect of shifting the saccade goal. Vision Research, 22, 353-364.

Deubel, H., Wolf, W., \& Hauske, G. (1984). The evaluation of the oculomotor error signal. In A. G. Gale \& F. Johnson (Eds.), Theoretical and applied aspects of eye movement research (pp. 55-62). Amsterdam: Elsevier, North-Holland.

Downing, P. E., \& DodDS, C. M. (2004). Competition in visual working memory for control of search. Visual Cognition, 11, 689-703.

DunCan, J., \& Humphreys, G. W. (1989). Visual search and stimulus similarity. Psychological Review, 96, 433-458.

Franconeri, S. L., Hollingworth, A., \& Simons, D. J. (2005). Do new objects capture attention? Psychological Science, 16, 275-281.

Frost, D., \& PöPpel, E. (1976). Different programming modes of human saccadic eye movements as a function of stimulus eccentricity: Indications of a functional subdivision of the visual field. Biological Cybernetics, 23, 39-48.

Henderson, J. M., Brockmole, J. R., Castelhano, M. S., \& Mack, M. (2007). Visual saliency does not account for eye movements during search in real-world scenes. In R. van Gompel, M. Fischer, W. Murray, \& R. Hill (Eds.), Eye movements: A window on mind and brain (pp. 537-562). Oxford: Elsevier.

Henderson, J. M., \& Hollingworth, A. (1999). The role of fixation position in detecting scene changes across saccades. Psychological Science, 10, 438-443.

Henderson, J. M., \& Hollingworth, A. (2003). Eye movements and visual memory: Detecting changes to saccade targets in scenes. Perception \& Psychophysics, 65, 58-71.

Hoffman, J. E., \& Subramaniam, B. (1995). The role of visual attention in saccadic eye movements. Perception \& Psychophysics, 57, 787-795.

Hollingworth, A. (2004). Constructing visual representations of natural scenes: The roles of short- and long-term visual memory. Journal of Experimental Psychology: Human Perception \& Performance, 30, 519-537.

Hollingworth, A., Richard, A. M., \& LuCK, S. J. (2008). Understanding the function of visual short-term memory: Transsaccadic memory, object correspondence, and gaze correction. Journal of Experimental Psychology: General, 137, 163-181.

HYUN, J.-S., \& LUCK, S. J. (2007). Visual working memory as the substrate for mental rotation. Psychonomic Bulletin \& Review, 14, 154-158.

IRwIN, D. E. (1992). Memory for position and identity across eye movements. Journal of Experimental Psychology: Learning, Memory, \& Cognition, 18, 307-317.

IRWIN, D. E., \& ANDREWs, R. V. (1996). Integration and accumulation of information across saccadic eye movements. In T. Inui \& J. L. McClelland (Eds.), Attention and performance XVI: Information integration in perception and communication (pp. 125-155). Cambridge, MA: MIT Press, Bradford Books.

IRWIN, D. E., \& GoRDON, R. D. (1998). Eye movements, attention, and trans-saccadic memory. Visual Cognition, 5, 127-155.

Kapoula, Z. (1985). Evidence for a range effect in the saccadic system. Vision Research, 25, 1155-1157.

Kapoula, Z., \& Robinson, D. A. (1986). Saccadic undershoot is not inevitable: Saccades can be accurate. Vision Research, 26, 735-743.

LAND, M. F., \& HaYhoE, M. (2001). In what ways do eye movements contribute to everyday activities? Vision Research, 41, 3559-3565.

LuCK, S. J. (2008). Visual short-term memory. In S. J. Luck \& A. Hollingworth (Eds.), Visual memory (pp. 43-86). New York: Oxford University Press.

LuCK, S. J. (2009). The spatiotemporal dynamics of visual-spatial attention. In F. Aboitiz \& D. Cosmelli (Eds.), From attention to goaldirected behavior: Neurodynamical, methodological, and clinical trends (pp. 51-66). Berlin: Springer.

Luck, S. J., \& Vogel, E. K. (1997). The capacity of visual working memory for features and conjunctions. Nature, 390, 279-281.
Matin, E. (1974). Saccadic suppression: A review and an analysis. Psychological Bulletin, 81, 899-917.

McPeeK, R. M., \& Keller, E. L. (2001). Short-term priming, concurrent processing, and saccade curvature during a target selection task in the monkey. Vision Research, 41, 785-800.

McPeek, R. M., \& Keller, E. L. (2002). Superior colliculus activity related to concurrent processing of saccade goals in a visual search task. Journal of Neurophysiology, 87, 1805-1815.

Moore, T., \& Armstrong, K. M. (2003). Selective gating of visual signals by microstimulation of frontal cortex. Nature, 421, 370-373.

Moore, T., \& Fallah, M. (2004). Microstimulation of the frontal eye field and its effects on covert spatial attention. Journal of Neurophysiology, 91, 152-162.

Moore, T., Tolias, A. S., \& Schiller, P. H. (1998). Visual representations during saccadic eye movements. Proceedings of the National Academy of Sciences, 95, 8981-8984.

Müller, J. R., Philiastides, M. G., \& Newsome, W. T. (2005). Microstimulation of the superior colliculus focuses attention without moving the eyes. Proceedings of the National Academy of Sciences, $102,524-529$

Olivers, C. N. L. (in press). What drives memory-driven attentional capture? The effects of memory type, display type, and search type. Journal of Experimental Psychology: Human Perception \& Performance.

Olivers, C. N. L., Meijer, F., \& Theeuwes, J. (2006). Feature-based memory-driven attentional capture: Visual working memory content affects visual attention. Journal of Experimental Psychology: Human Perception \& Performance, 32, 1243-1265.

Richard, A. M., \& Hollingworth, A. (2008). Strategic control of visual short-term memory during scene viewing. Paper presented at the Annual Meeting of the Vision Sciences Society, Naples, FL. Retrieved March 12, 2009, from www.psychology.uiowa.edu/faculty/ hollingworth/publications.html.

Richard, A. M., Luck, S. J., \& Hollingworth, A. (2008). Establishing object correspondence across eye movements: Flexible use of spatiotemporal and surface feature information. Cognition, 109, 66-88.

SATO, T. R., \& Schall, J. D. (2003). Effects of stimulus-response compatibility on neural selection in frontal eye field. Neuron, 38, 637-648.

SCHALL, J. D. (2002). The neural selection and control of saccades by the frontal eye field. Philosophical Transactions of the Royal Society B, 357, 1073-1082.

Schmidt, B. K., Vogel, E. K., Woodman, G. F., \& LucK, S. J. (2002). Voluntary and automatic attentional control of visual working memory. Perception \& Psychophysics, 64, 754-763.

Sheinberg, D. L., \& Logothetis, N. K. (2001). Noticing familiar objects in real world scenes: The role of temporal cortical neurons in natural vision. Journal of Neuroscience, 21, 1340-1350.

Soto, D., Heinke, D., Humphreys, G. W., \& Blanco, M. J. (2005). Early, involuntary top-down guidance of attention from working memory. Journal of Experimental Psychology: Human Perception \& Performance, 31, 248-261.

Soto, D., \& Humphreys, G. W. (2008). Stressing the mind: The effect of cognitive load and articulatory suppression on attentional guidance from working memory. Perception \& Psychophysics, 70, 924-934.

Soto, D., Humphreys, G. W., \& HeInKe, D. (2006). Working memory can guide pop-out search. Vision Research, 46, 1010-1018.

Woodman, G. F., \& LUCK, S. J. (2007). Do the contents of visual working memory automatically influence attentional selection during visual search? Journal of Experimental Psychology: Human Perception \& Performance, 33, 363-377.

WoOdMan, G. F., LuCK, S. J., \& SCHALL, J. D. (2007). The role of working memory representations in the control of attention. Cerebral Cortex, 17, 118-124.

YANTIS, S., \& Jonides, J. (1984). Abrupt visual onsets and selective attention: Evidence from visual search. Journal of Experimental Psychology: Human Perception \& Performance, 10, 601-621.

ZHANG, W., \& LUCK, S. J. (2008). Discrete fixed-resolution representations in visual working memory. Nature, 453, 233-235.

\section{NOTES}

1. We consider the terms visual working memory and visual shortterm memory as describing the same set of processes. Recent evidence that visual working memory is strategically controlled in the service of 
current task demands (Hyun \& Luck, 2007; Richard \& Hollingworth, 2008) leads us to prefer the former term (see Luck, 2008, for a detailed discussion).

2. In this view, the maintenance of visual features in VWM and the feature-based attentional selection of objects during search depend on a common set of perceptual representations, and thus interact dynamically.

3. We define a corrective saccade functionally, as a secondary saccade that corrects for the error generated by an inaccurate primary saccade. There exists a large literature on corrective saccades. The vast majority of this literature concerns gaze correction when there is direct visual information specifying the location to which the corrective saccade should be generated (i.e., visually guided gaze corrections, as opposed to the memory guided gaze corrections in the present study). Much of the existing work on corrective saccades has used the double-step paradigm introduced by Becker and Jürgens (1979). In this paradigm, a saccade target object is shifted immediately prior to the primary saccade, so that its new location is visible before the initiation of the primary saccade. In this case, a corrective saccade is often computed in parallel with the primary saccade, supporting exceedingly rapid gaze correction to the target's new location (Becker \& Jürgens, 1979) and even causing curvature of the primary saccade toward the new location (McPeek \& Keller, 2001). In addition, most studies examining corrective saccades have used single saccade target objects (Becker, 1972; Deubel, Wolf, \& Hauske, 1982; Kapoula \& Robinson, 1986). With a single object, there is no target ambiguity when the eyes land, and gaze correction can be based on direct perception of the target location after the saccade. Our method differs from the above work on visually guided corrective saccades, because the rotation of the array occurs during, rather than before, the primary saccade, and a corrective saccade cannot be preprogrammed. Corrective saccade latencies in the present method are therefore significantly longer than those found in the double-step paradigm. In addition, the presence of multiple objects when the eyes land necessitates the use of memory to select the appropriate location for the corrective saccade. This type of memory-guided gaze correction has been investigated in only a handful of studies (Deubel, Wolf, \& Hauske, 1984; Hollingworth et al., 2008; Richard et al., 2008). Yet memory-guided corrective saccades are likely to be much more frequent in real-world behavior than are the types of visually guided corrective saccades typically studied in the literature. A situation analogous to the double-step paradigm would occur only in the very rare circumstance that a saccade target object moves precisely when a primary saccade has been computed but has yet to be initiated. Given the complexity of natural environments, it would certainly be a rare occurrence that the saccade target object was the only object visible after the saccade.

4. This 40-msec slowing may also reflect the simple fact that multiple items were near the landing point of the initial eye movement in the condition that tested the use of VWM in gaze corrections. Only a single item was present on the screen in the no-memory control condition, reducing low-level motor competition. Thus, the use of memory per se may have added significantly less than $40 \mathrm{msec}$ to the corrective saccade latency.

5. The distances of objects from the landing position of the saccade can influence secondary, corrective saccades, with an object closer to the landing position more likely to be selected as the target of the correction than one further from the landing position. However, when memory can serve to distinguish objects near the landing position, the influence of a memory match is much stronger than is the influence of distance. Corrective saccades are more likely to be directed to the original target object (matching memory) than to the distractor, even when the eyes land significantly closer to the distractor than to the target (Hollingworth et al., 2008).

6 . In this paradigm, although participants cannot see the rotation during the saccade, they can often infer, after the saccade, that the array rotated. However, this inference requires memory for the colors of the disks, and this inferential process appears to play little or no role in the fast and automatic gaze corrections (Hollingworth et al., 2008).

7. It is possible, in theory, that participants could shift attention only when the color of the distractor exactly matched the color being maintained in VWM. However, this would require that the participants'VWM representations be so precise that they could inhibit a shift of attention to slightly different shades of the same color. If they could do this, they presumably would have a sufficiently good representation of the color to perform the memory task without such an exotic strategy (especially given that VWM representations of color retain their precision for at least 4 sec; Zhang \& Luck, 2008).

(Manuscript received August 25, 2008; revision accepted for publication December 23, 2008.) 